\title{
Direct imaging and manipulation of ionic diffusion in mixed
}

\section{electronic-ionic conductors}

J. Op de Beeck*

IMEC, Kapeldreef 75

KU Leuven, Department of Physics and Astronomy, Celestijnenlaan 200D, B-3001 3001

Belgium

Nouha Labyedh

IMEC, Kapeldreef 75

KU Leuven, Department of Microbial and Molecular Systems, Celestijnenlaan 200D 3001

Belgium

Dr. A. Sepúlveda, Dr. V. Spampinato, Dr. A. Franquet, Dr. T. Conard

IMEC, Kapeldreef 75

3001

Belgium

Prof. Dr. P. M. Vereecken

IMEC, Kapeldreef 75

KU Leuven, Department of Microbial and Molecular Systems, Celestijnenlaan 200D 3001

Belgium

Dr. U. Celano*

IMEC, Kapeldreef 75

3001

Belgium

Corresponding authors: Jonathan.OpdeBeeck@imec.be, Umberto.Celano@imec.be

Keywords: Li-ion kinetics, 3D thin-film batteries, ASB, C-AFM, SIMS 
Next generation Li-ion batteries require improved energy densities, power output and safety to satisfy the demands of emerging technologies. All solid state (ASB) 3D thin-film batteries based on nanoionics are considered as frontrunners to enable all this. In order to facilitate the introduction of this new architecture, a homogeneous electrochemical activity and a high ionic diffusivity of the electrodes is key. However, nanometer-resolved techniques to probe structural, electrical and electrochemical properties of the battery components are still limited. Here we propose a study that combines conductive atomic force microscopy (C-AFM) and secondary ion mass spectrometry (SIMS) for the structural and electrical characterization. In addition, a novel concept called ion-modulated C-AFM (imC-AFM) is introduced to sense also the electrochemical activity of ions in confined volumes. Using the aforementioned methodologies, $\mathrm{Li}_{\mathrm{x}} \mathrm{Mn}_{2} \mathrm{O}_{4}$ thin films cathodes are studied observing: (1) a direct correlation between electrical conductivity and local chemistry. (2) A non-uniform Li-ion electrochemical activity (i.e. ionic conductivity) on the cathode's surface with a clear enhancement in grain boundaries (GBs). Finally, (3) imC-AFM observes a high volume expansion associated with high Li incorporation. This work introduces a novel pathway for the rapid analysis of materials to be used in ASB.

\section{Introduction}

The three-dimensional thin film all solid state battery is an emerging technology tackling three main demands of modern society on batteries. ${ }^{1,2}$ First of all, the absence of flammable liquid, replaced by a solid state (SS) electrolyte, improves the safety. Secondly, the use of thin films is beneficial in order to increase the charge/discharge rate i.e. the power, compensating for the relatively limited ionic and electronic conductivity of oxide materials. Finally, through the 3D patterning, the increase in effective surface area maximizes the battery. The latter compensates for the nominal loss in capacity introduced by the reduction of films' thicknesses, resulting in performance that exceeds actual planar thin film ASB design. ${ }^{3}$ However, a design based on 3D thin films presents various challenges for the deposition and integration of different materials. 
Variations in the material stability and chemical composition of the insertion electrodes leads to a non-uniform cell utilization with major efficiency losses. ${ }^{4}$ Furthermore, local thermodynamic and kinetic variations are leading to inhomogeneous electrochemical activity across the final battery stack. For example, structural inhomogeneity and low diffusion rate of the electrodes can ultimately introduce non-uniform cathode utilization. $[2,3]$

Typically, galvanostatic studies and other relatively large size analysis setups are applied to understand bulk characteristics such as the Li-ion kinetics, phase transitions and diffusion coefficients.[4] This represents a major limitation when addressing local scale, e.g. micro- or nanoscale, phenomena owing to the averaged nature of the measured information. A central challenge is the development and assessment of nm-resolved characterization techniques suitable for the analysis of electrical and nanoionic phenomena.[5] For this reason, in contrast with traditional methods, here we use a nanosized electrode, i.e. the tip of an atomic force microscope, thus sensing a corresponding electrochemical response in a confined volume with nanometer resolution. Scanning probe microscopy (SPM) has been already proposed to study Li-ion electrodes.[6-11] Notably, developed to probe ionic processes using AFM, electrical strain microscopy (ESM)[11] and first order current-voltage (FORC-IV) [8] have been proposed. While the first has provided a significant contribution to the understanding of bias induced local Li-ion transport, the second has to our knowledge not yet been applied to lithium compounds although suggested by E. Strelcov and co-authors.[10] Both techniques provide complementary response factors, electrical hysteresis and volume expansion. However, they still require a relatively complex implementation, especially for the high speed data acquisition hardware used in band excitation method.[12] To date, a combined analysis platform that provides structural, chemical, electrical and electrochemical information with nm lateral resolution is still missing. Here we propose the combination of C-AFM and SIMS in a single apparatus, which provides a sub- $\mu \mathrm{m}$ resolved analysis-setup for the direct correlation of structural and electrical properties with the chemical composition of Li-ion insertion electrodes. 
However, with the reduction of the films thickness and the increase in density of 3D architectures, the nanoionic character of the system dominates the bulk properties of the materials impacting the battery operation strongly.[13] For this reason, starting from a conventional C-AFM, we introduce here a new mode, ion-modulated C-AFM (imC-AFM) providing information on the ionic activity and corresponding volume expansion with sub-50 nm lateral resolution.

\section{Results and discussion}

\subsection{Combined electrical and structural analysis}

For its competitive capacity of $1.24 \mathrm{Ah} / \mathrm{cm}^{3}$ and widespread usage in rechargeable Li-ions battery technology, the $\mathrm{MnO}_{2}$ cathode shows great promise also for ASB applications. The spinel crystal structure, that allows Li-ion migration through a 3D network of mobile channels, makes this electrode a strong candidate also as cathode material for 3D thin-film all solid state batteries.[14] Figure 1 shows the two materials used as model systems in this work. We focus our analysis on electrodeposited and RF-sputtered lithium manganese oxide (LMO) in Figure 1a,b respectively. The two deposition techniques result in a different surface quality as visible by the SEM analysis of the morphology. However, both films show excellent cyclability and performance as previously evaluated by galvanostatic charge and discharge measurements.[15] 

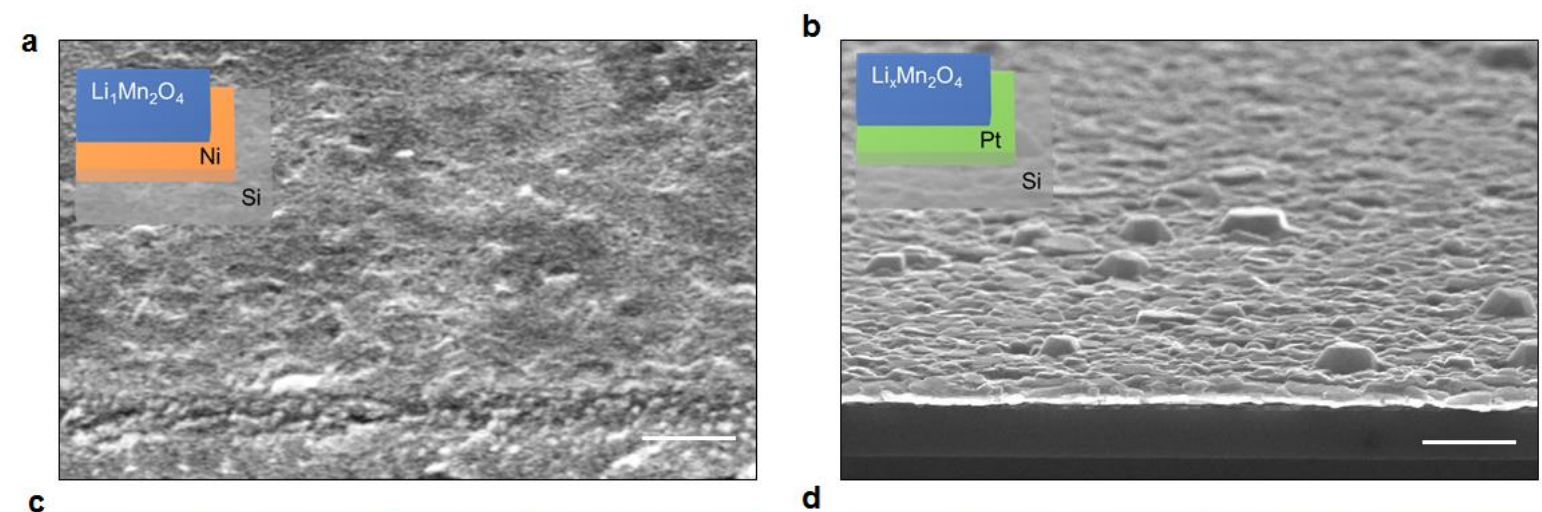

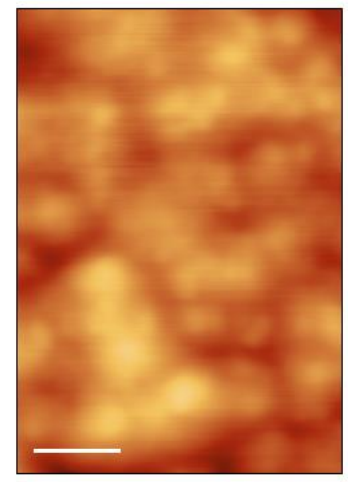

0

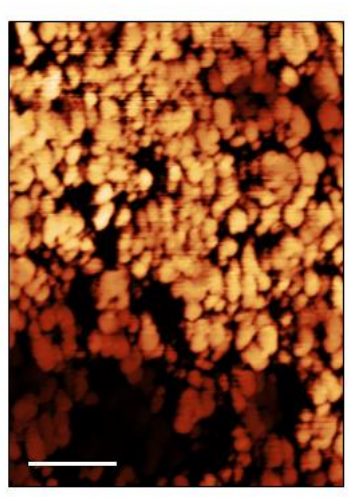

0

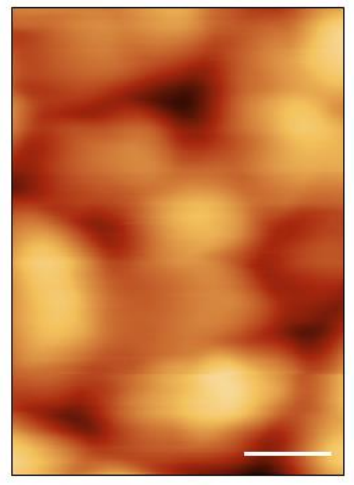

0

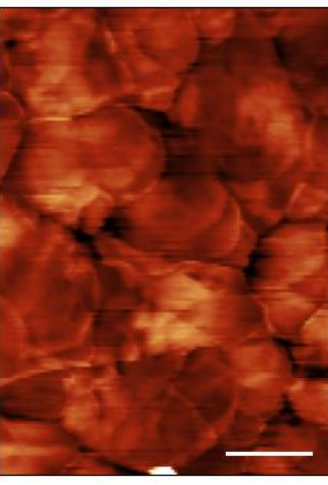

$0 \rightleftharpoons 8 \mathrm{nA}$

Figure 1. Lithium manganese oxide layers surface comparison. (a) SEM image of the top surface for a $260 \mathrm{~nm}$ thick electrodeposited LMO. (b) SEM image of a $100 \mathrm{~nm}$ thick RFsputtered LMO. A clear granularity with increased roughness is visible for this sample. Note, both films are deposited on a metal current collector. (c) C-AFM morphology (left) and current map (right) for the electrodeposited sample (scale bar $200 \mathrm{~nm}$ ) in which the bias is applied between the conductive tip and the current collector $(3 \mathrm{~V})$. (d) The same is repeated for the RFsputtered LMO (scale bar $200 \mathrm{~nm}$ ).

Figure 1c,d shows the topography and current maps of the two LMO samples measured by CAFM under ambient conditions. The electrodeposited LMO ( $260 \mathrm{~nm}$ thick $)$ shows the presence of crystalline grains with an estimated surface roughness (RMS value) of 3,02 nm over an area of $2 \times 2 \mu \mathrm{m}^{2}$ as in Figure 1c. A detailed small scale analysis of the clusters reveals the presence of small grains with an average grain diameter of ca. $70 \mathrm{~nm}$ (Figure 1c right). These are perfectly visible in the current map of C-AFM as small islands with highly conductive 
core and insulating grain boundaries. Differently, the RF-sputtered film (Figure 1d) has larger grains ca. $290 \mathrm{~nm}$ size leading to an increased surface roughness (RMS value) estimated as $27,2 \mathrm{~nm}$ over an area of $2 \times 2 \mu \mathrm{m}^{2}$. The level of conductivity observed is higher for the RFsputtered LMO, although this is expected due to the reduced sample's thickness. In this case it is more difficult to assess the electrical conductivity of the grain boundary without ambiguity, as both highly conducting and isolating boundaries are observed. Two possible reasons could explain the observation of a highly conductive boundary. One reason could be the local defective nature of the boundary which can lead to a lower resistive path. A second reason could be the AFM tip sidewall interacting with both sides of the boundary inducing a larger contact area fort he current to flow.

\subsection{Combined electrical and chemical analysis}

The conductive domains observed in the current maps of Figure 1c,d suggest that the chemical inhomogeneity of the surface determines a spatial distribution of the local conductivity. To disentangle the correlation between the chemical phase and conductivity, C-AFM and SIMS are combined on the same location. In essence, inside a single apparatus a movable stage positioned the same area with sub- $\mu \mathrm{m}$ precision between the two techniques. Therefore, we use first C-AFM (electrical properties) and afterwards SIMS (chemical composition) allowing a comparative study of the same area with nanometric precision. Figure 2 shows the results obtained for the electrodeposited and RF-sputtered LMO. For each sample we scan with CAFM (tip negatively biased, 5-8 V) an area of size $15 \times 15 \mu \mathrm{m}^{2}$. This is followed by the static SIMS analysis in Fast Imaging mode (ca. $100 \mathrm{~nm}$ lateral resolution), which is performed with a $\mathrm{Bi}_{3}$-cluster beam in the area previously studied by C-AFM. Figure $2 \mathrm{a}-\mathrm{c}$ show the case of the electrodeposited $\mathrm{Li}_{x} \mathrm{Mn}_{2} \mathrm{O}_{4}$, where the net contrast in the current map (Figure 2b) indicates a non-uniform conductive behavior. Figure $2 \mathrm{c}$ shows the lithium distribution on the same area of 
the current map, thus revealing the chemical nature of the electrical-contrast with high sensitivity and high lateral resolution (ca. $100 \mathrm{~nm}$ ). A clear correlation is observed between the current map and the chemical lithium map, with highly conductive regions associated with a higher content of Li on the surface (brighter spots on the chemical map). Vice versa, regions poor in lithium are also relatively less conductive and almost insulating. Our observations reveal that for the electrodeposited LMO the highly lithiated domains are very localized on the micrometer scale. It is worth noting that C-AFM does not make differences on electronic and ionic contribution in the sensed current, measuring the sum of the two. The latter will be shown in section 2.4 in which acquired I-V characteristic indicate a shift due to an ionic contribution with no modification on the transport characteristics. It is therefore not obvious to observe such a high correlation between conductivity and $\mathrm{Li}$ for a material that is supposedly a mixed ionicelectronic conductor. Figure 2d-f shows the C-AFM/SIMS analysis of the RF-sputtered $\mathrm{Li}_{1} \mathrm{Mn}_{2} \mathrm{O}_{4}$. Here the C-AFM analysis indicates the presence of highly conductive nonuniformities extending in range $1-3 \mu \mathrm{m}$. In agreement with the electrodeposited LMO, also here a higher electrical conductivity is generally observed in correspondence of Li-rich regions (Figure 2d-f). In this case, a relatively more homogeneous presence of lithiated domains is visible (Figure 2f). It is worth mentioning that, especially in the case of Figure 2d-f, exceptions in which the SIMS and C-AFM data do not agree can be found. However, this is to be expected as the surface has been most likely modified during the C-AFM data acquisition as elaborated on in section 2.3 (Figure 3). 
a

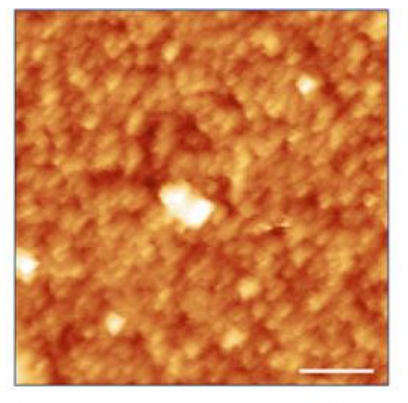

0

d

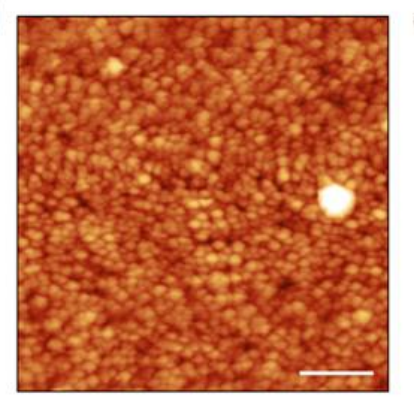

0
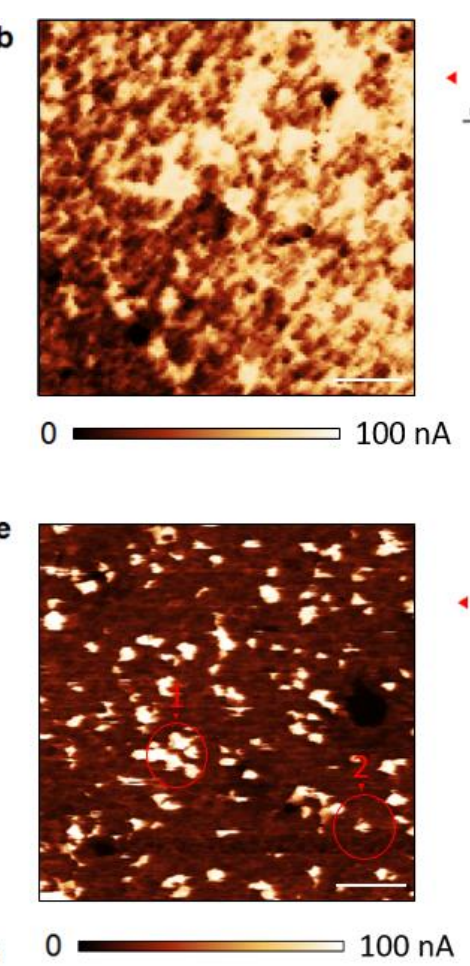
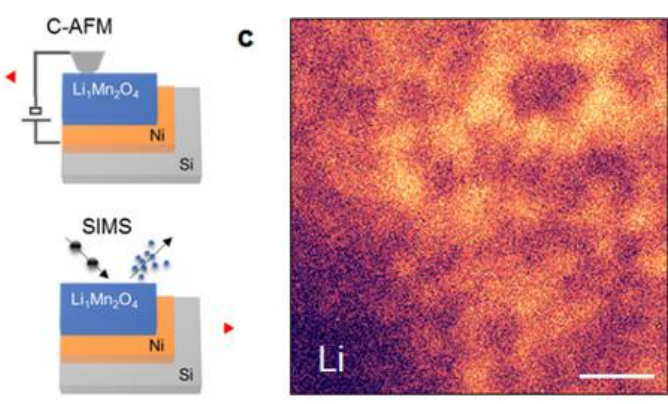

$0 \longrightarrow 80$

Ion counts

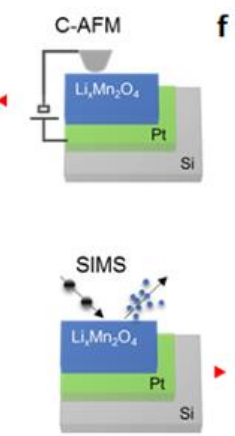

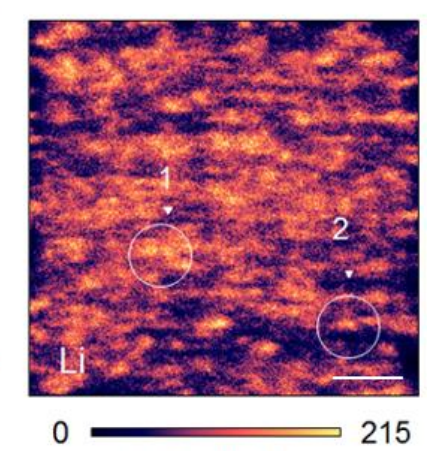

Ion counts

Figure 2. Combined study of LMO by C-AFM and SIMS. (a) C-AFM morphology and (b) current map of a $15 \times 15 \mu \mathrm{m}^{2}$ area of the electrodeposited LMO (scale bar $3 \mu \mathrm{m}$ ). (c) The SIMS mapping of the same area shows a phase separation with different lithiation levels at the micrometer scale. Highly lithiated regions show significantly more current as visible by the good correlation of Li concentration and high C-AFM current. (d) Morphology of the surface for the RF-sputtered LMO and (e) associated current map (scale bar $3 \mu \mathrm{m}$ ). (f) Despite the higher roughness visible in the AFM, the SIMS analysis indicates enhanced homogeneity of the lithium spatial distribution on the surface. Note in the insets, a good correlation between highly lithiated areas and low electrical resistance is observed. For both samples our results indicate that we are not in a regime of maximal cathode utilization.

Clearly, a homogeneous material phase is desired in Li-ion batteries as it dictates the local electrochemical activity and ensures maximum cathode utilization. The maps shown in Figure 2 indicate that a large variation exist in the spatial distribution of material phases and local conductivity in the sub- $\mu \mathrm{m}$ scale. The observed non-uniformities are likely due to a different 
local stoichiometry, crystalline orientation and defects. In the case of electrodeposited LMO (Figure 2a-c) the highly lithiated domains are clearly spatially separated from the lithium poor, lowly conductive regions. As the oxidation of manganese benefits during battery charge operation from the high electrical conductivity in the lithium rich regions, it is highly desired that these regions are easily accessible and interconnected. Therefore, in this case the electron transport is significantly hampered as the highly conductive regions are not immediately accessible for electron transport. Moreover, the electrochemical activity within this cathode will certainly not be homogeneous and maximum cathode utilization is likely not achieved. On the contrary, the RF-sputtered sample (Figure 2d-f) provides a more homogeneous sample with accessible and interconnected highly conductive regions. Notably, Li-ion insertion electrodes generally have a rough surface topography and contain many grains (see Figure $2 b, e$ ). Therefore they are prone to induce artifacts in the SIMS measurement. The origin of possible artifacts is a difference in secondary ion yield because the angle of incidence changes with respect to the surface normal. The analysis will therefore appear distorted because of the samples morphology.[16] In this work, we repeatedly inspected the morphology of the sample after the ionic sputtering process in order to detect unexpected surface changes. Additionally we can also rely on the current map to detect any anomalies. The elemental analysis, including other elements and the in-depth SIMS profiles of the two samples, are available in supporting information Figure S1. It is worth to note that, despite the good correlation observed for both samples between lithium concentration and high conductivity (inset Figure 2e,f), the C-AFM measurement does not probe the electrical properties of the sample, but rather the sum of the electronic and ionic current which is flowing in the tip-sample system. It is not possible to decouple the electronic and the ionic contribution losing important information about the lithium diffusion or electrochemical activity. 


\subsection{Bias-induced modulation of lithium}

The presence of mobile ions in thin films allows a biased tip to trigger a field-induced ion migration and reactions, thus altering the conductive properties of the layer. The latter has been previously demonstrated by Kumar et al. in yttrium-stabilized zirconia (YSZ) and E. Strelcov et al. in a Ca-substituted bismuth ferrite (Ca-BFO) as a method for the nanomanipulation of oxygen vacancies in mixed ionic-electronic conductors underneath a conductive AFM tip.[8,17,18] Consistently, we can induce a local change in conductivity in our films by alternating the tip polarity. Figure 3a shows this effect by comparing the local conductivity inside four different areas of the $\mathrm{Li}_{1} \mathrm{Mn}_{2} \mathrm{O}_{4}$ that have been stressed with different voltage polarity. By comparing the pristine state with the local conductivity change induced in the stressed areas, a suppression of current is visible by scanning with a tip positively biased (Figure 3a bottom dashed boxes), while this is not the case reversing the polarity (Figure 3a top dashed boxes). However, in the latter case the expected conductivity increase due to the attraction of $\mathrm{Li}$ ions to the surface is minimal as also shown later in section 2.4 (Figure 4a). Note, in both cases we do not observe any morphological changes in the stressed areas because of the inert ambient (high vacuum $10^{-5}$ mbar). On the contrary, measurements performed in air can induce morphological surface changes owing to undesired side reactions occurring between tip and sample (See supporting information Figure S2). The effect of Figure 3a can be qualitatively explained by the local accumulation and depletion of the $\mathrm{Li}$ ions on the tip passage. Due to their positive charge, $\mathrm{Li}$ ions will accumulate on the top surface while scanning the sample with a negatively biased tip (Figure 3a). On the contrary the opposite effect is induced by scanning the tip positively biased. In other words, with the AFM tip it is possible to apply voltage stimuli that initiate the internal redistribution of $\mathrm{Li}$ ions by intercalation and de-intercalation (Figure 3b). Thanks to the sensing capability of C-AFM and SIMS, we can probe directly the change in Li concentration by a chemical mean. The area previously stressed with a negatively biased tip $(-8 \mathrm{~V})$ is probed by SIMS in Figure 3c. A strong lithium background signal (i.e. higher 
amount of $\mathrm{Li}$ ) is observed compared to the pristine material. These results clearly indicate the capability of the tip to locally induce the field-induced diffusion of Li ions. It is worth noting that no attraction of $\mathrm{Na}^{+}$or $\mathrm{K}^{+}$was observed, as their signal did not show any contrast with the pre-scanned area (see Supporting Information Figure S1 d,e). In light of the clear presence of $\mathrm{Na}^{+}$or $\mathrm{K}^{+}$in the chemical profiles, and considering the high chemical sensitivity of SIMS, this is likely the result of their relative lower mobility inside the LMO. Importantly, while the observation in Figure $3 \mathrm{c}$ involves a relatively large area $\left(15 \mathrm{x} 15 \mu \mathrm{m}^{2}\right)$, thanks to the strong confinement of the electric field under the tip, a smaller volume (ca. 2-3 times the tip radius) can be probed by a point-contact voltage stress.[19] 

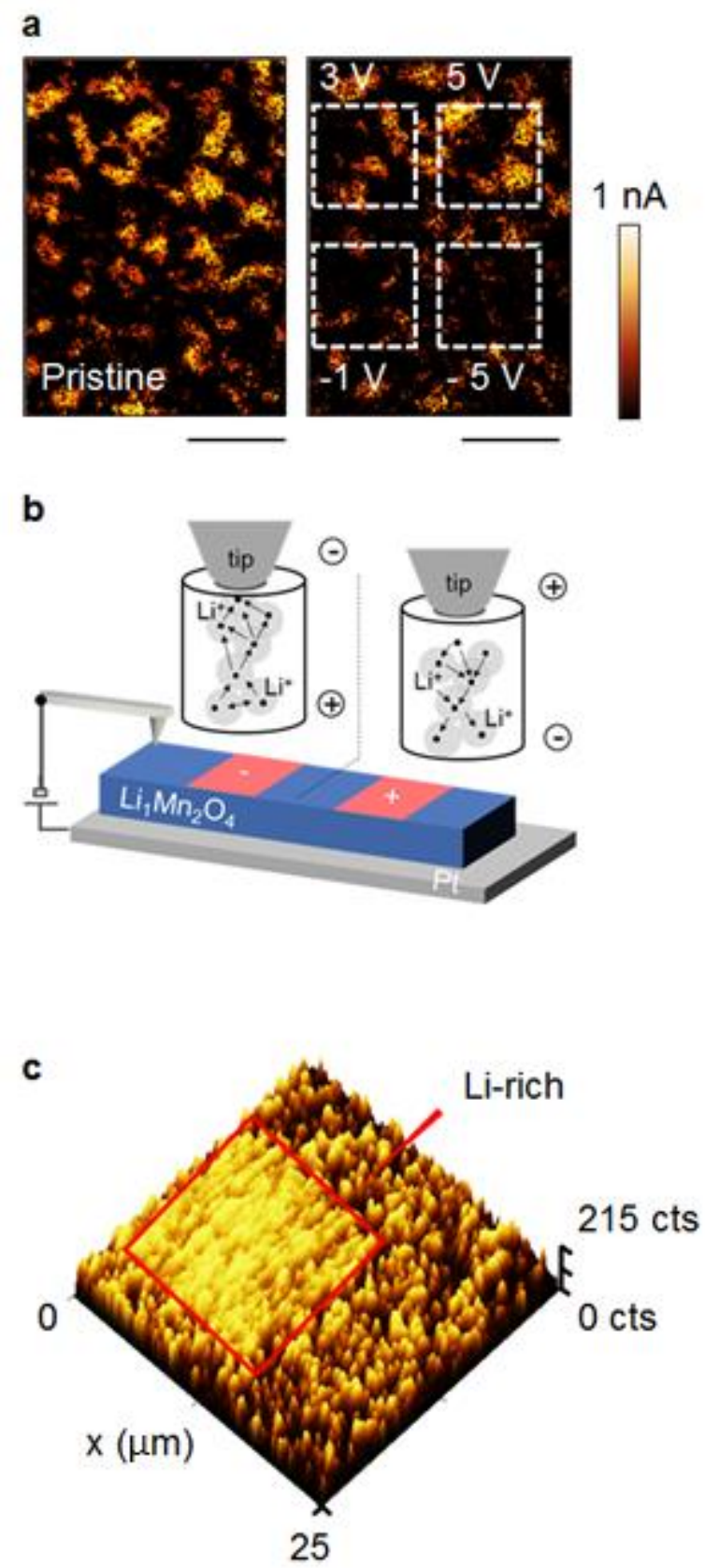

Figure 3. Tip-induced ionic migration under different bias polarity. (a) C-AFM current maps before and after a pristine LMO region has been stressed in four regions (dashed boxes). The top row of squares represents the accumulation of Li-ions, while the bottom row indicates their depletion. (b) Schematic representation of the tip-induced electric field-activated Li ions accumulation/depletion process. (c) The effect of a prolonged AFM scan (8 V) is visible by 
means of SIMS, providing the chemical evidence of the effective ionic migration and lithium redistribution within the stack.

While Figure $3 \mathrm{c}$ shows the chemical composition of the stressed area, also the electrical conductivity of the volume probed by the tip undergoes a significant change depending on the lithium concentration (Figure 2c,f). This effect has been already observed by others in different Li-ion compounds both at the nanoscale[18,20,21]' and macroscale.[22] The change in conductivity is strongly related to the impact of the different stoichiometry due to the ion diffusion on the local band structure. In essence, by continuous DC cycling the resistance of the confined volume beneath the tip is modified (inset Figure 3a). Accordingly, a strongly varying current-voltage (I-V) characteristic is visible (Figure 4a) by applying a saw-tooth DC waveform (inset) to the tip-sample system. It is worth to mention that as the tip bias changes over time, hysteretic I-V curves are visible with a consistent and reproducible behavior. The ionic nature of this effect, becomes clear by studying the dependency of the I-V hysteretic opening on the bias frequency as shown in Figure $4 \mathrm{~b}$. Depending on the rate at which the bias is swept, the amount of the hysteresis changes, i.e. increasing or reducing respectively with slow and high rates. This is consistent with the ionic diffusion time of the mobile species migrating in the small probed volume. In other words, at high frequency only the electronic contribution of the current is sensed. Furthermore, a large ion diffusion coefficient in the local region will lead to a larger change in Li concentration and consequently a larger hysteresis.

\subsection{Ion-modulated C-AFM}

\subsection{1. imC-AFM principles}

The observations above provide the pathway to develop a methodology based on C-AFM, which extends the sensing capability to the electrochemical response of the probed volume using hysteretic I-V curves. Inspired by the work on (ESM)' of Balke et al.[23] and Jesse et al.[11], and closely related to the FORC-IV[8] method, ion-modulated C-AFM is a 
straightforward and easily implemented approach based on the local nanomanipulation of lithium ions underneath a AFM tip. The ability to manipulate the ions underneath the AFM tip has been demonstrated and proven by AFM-SIMS in Figure 3 which is the foundation of ionmodulated C-AFM. In practice, after scanning the sample with C-AFM we collect multiple I$\mathrm{V}$ curves in a spatially resolved grid of points (typical spaced tens of nm to limit the crosstalk). The I-Vs are acquired with the tip negatively biased, thus resulting in a net attraction of positive mobile Li ions which changes the local conductivity, inducing visible hysteresis as in Figure 4a. The hysteresis amplitude is computed by integrating the area of the subsequent DC bias sweeps numerically. To establish a relationship between the hysteretic I-V loops and the ions electrochemical activity (i.e. ion diffusion), a Matlab script is used to extract the main parameters such as hysteresis loop area and tip-displacement. This generates a map which can be directly compared with the C-AFM measurement, while adding new information on the electrochemical activity in the scan area. Since electrochemical reactions and transport of ions are considered much slower compared to electrons transport (Figure 4b), by selecting a relatively slow voltage rate $(0.2-0.5 \mathrm{~Hz})$ we can directly probe the ionic or electronic contributions. Therefore, the loop opening of the I-V hysteresis can be directly related to the amount of lithium ions that are reacting in the volume sensed by the tip (i.e. ion diffusion). 

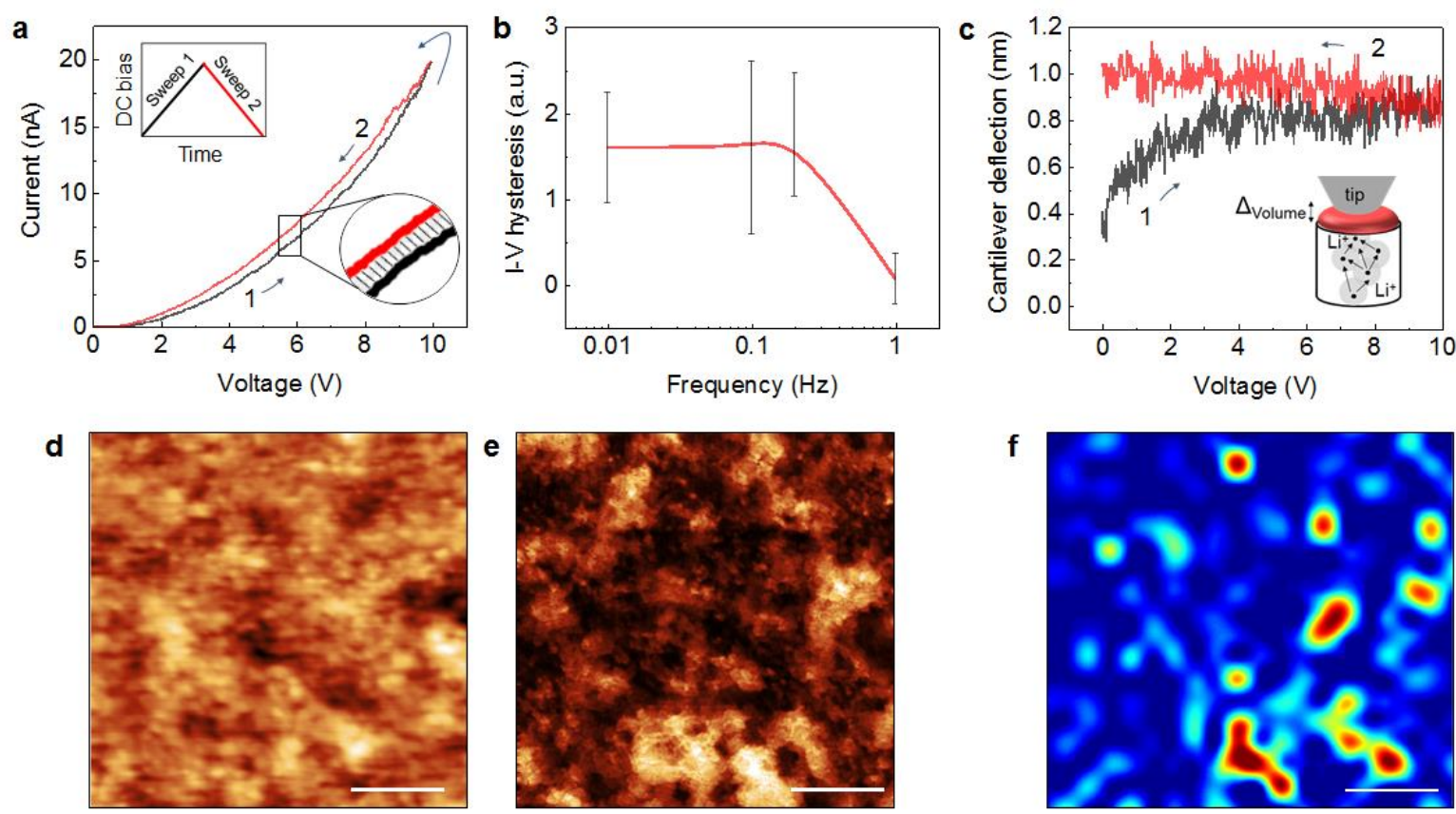

0

$25 \mathrm{~nm}$

0

$5 \mathrm{nA}$

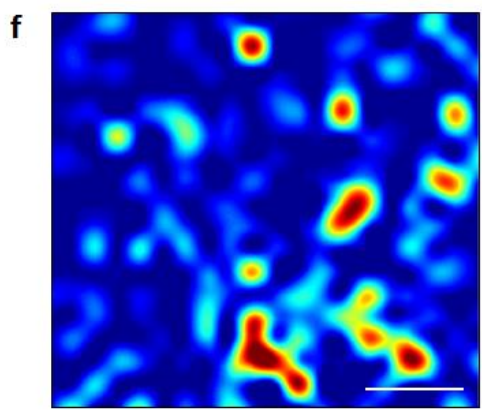

0

$2 \mathrm{nW}$

Figure 4. Ion modulated C-AFM mode of operation and contrast mechanisms. (a) Local IVcurve showing hysteretic behavior, with the increased conductivity visible on the backward voltage sweep. The insets shows the bias sweep applied with negative AFM-tip polarity and the corresponding electrical hysteresis area of the loop. (b) Dependency of the I-V hysteretic opening on the bias frequency. A significantly increase of the hysteresis in the I-V appears by lowering the sweep rate. (c) Cantilever deflection sensed during the hysteretic I-V curve in response to the volume expansion of the probed volume (inset). (d) imC-AFM morphology and (e) current map for the electrodeposited LMO (scale bar $1 \mu \mathrm{m})$. (f) The hysteretic loop opening represents the electrochemical activity in the area (acquired by voltage sweep $12 \mathrm{~V}$, sweep rate $0.5 \mathrm{~Hz}, 20 \times 20$ grid with $200 \mathrm{~nm}$ points spacing).

In addition, ion migration and mechanical deformation are inherently linked in most Li-ion electrodes (e.g. 6\% expansion for $\mathrm{MnO}_{2}$ ).[24] Therefore, by tracking the tip deflection during the I-V curve it is also possible to follow the local volume expansion/compression which are sensed by the AFM photodiode (Figure 4c). In this way imC-AFM differentiates itself from 
others methods as it generates understanding on electrical, ionic and structural changes in the material. Moreover, in contrast with ESM, imC-AFM is able to probe the volume expansion but does not depend on the latter for the ionic sensitivity. Therefore it extends the range of application also to zero strain materials, such as $\mathrm{Li}_{4} \mathrm{Ti}_{5} \mathrm{O}_{12}$ for which the volume expansion is not a suitable response factor.

Figure $4 c-f$ shows the results obtained by imC-AFM for the electrodeposited LMO. Here we add a new channel to the standard morphology and current maps, namely the spatially resolved mapping of the electrochemical activity on the surface. Conventional morphology and current maps (Figure 4,d,e) are complemented by the electrochemical map in Figure 4f. The latter is the interpolated 2D map obtained by the treatment of the dense spatial grid of I-V curves on the same area. The values reported in $\mathrm{nW}$ are the hysteretic amplitudes extracted from all the curves. The differences visible in Figure 4e,f indicate the various contributions of ionic and electronic current on the surface. This piece of information was previously missing as shown in Figure $2 \mathrm{e}, \mathrm{f}$.

It is worth noting that the phase transitions induced by the AFM-tip are not limited to those desired in the ideal device operation. Indeed, reversible Li-ion insertion and extraction associated with the reversible reduction and oxidation of the active transition metal (reversible conversion of $\mathrm{Mn}(\mathrm{IV})$ to $\mathrm{Mn}(\mathrm{III})$ in $\mathrm{LiMn}_{2} \mathrm{O}_{4}$ ) are limited to a small voltage range (for $\mathrm{LMO}$ between $2.6 \mathrm{~V}$ and $4.4 \mathrm{~V}$ vs. $\mathrm{Li}^{+} / \mathrm{Li}$ ). [25] Upon inducing a reversible material phase in the scanned area under the tip, galvanic coupling due to the difference in electrochemical potential with the neighboring area will quickly restore it to its initial Li-ion distribution in the case of a fully reversible phase transition. However, irreversible conversion reactions may occur outside of the reversible voltage window which are responsible for loss in battery capacity during, for example, battery over charging or over discharging.[24] The fact that an area with increased lithium content was measured with SIMS many minutes after the AFM scan (Figure 3c), 
indicates that indeed an irreversible phase was formed at those applied tip biases. Lowering of the tip bias until no longer a permanent lithiated phase is found could possibly identify the voltage range that corresponds to the reversible potential range.

\subsubsection{Ionic activity, grain boundaries and volume expansion}

Figure 5 shows the application of imC-AFM on the RF-sputtered LMO. In the area under study we can clearly distinguish two crystalline grains and a GB running in the middle of Figure 5b. Interestingly, the current map (Figure 5b) shows different conductivity in the two grains (G1 and G2 in Figure 5b) with lower current flowing in G1 compared to G2. By comparing the ionic and electronic contribution in Figure 5b,c it becomes clear that G2 shows a relatively weak electrochemical response, i.e. reduced hysteresis that is ionic activity. These results come as no surprise, considering that the crystallographic orientation of the grains strongly influences the performance of the cathode materials. Furthermore, the local saturation of lithium at the surface, leading to the occupation of available crystal sites for Li intercalation can possibly have an important role as well. This effect can therefore also explain why G2, with higher conductivity and therefore a higher Li concentration, has a lower electrochemical response compared to G1. 


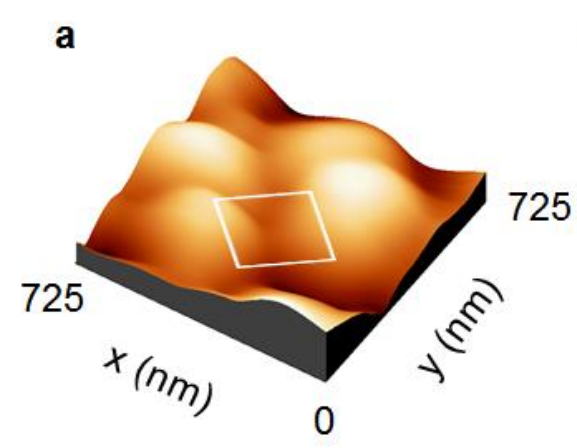

b

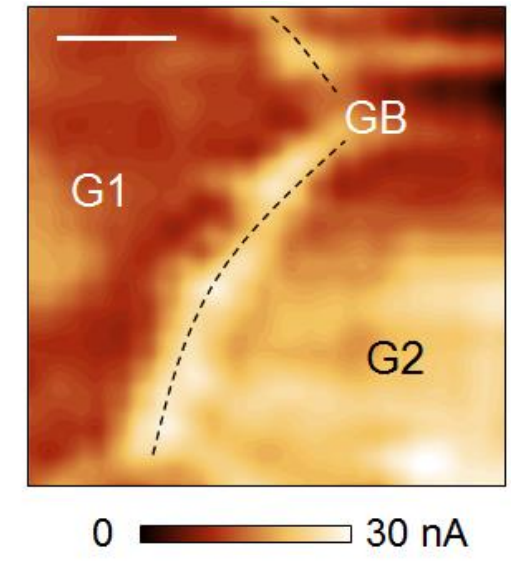

C

d
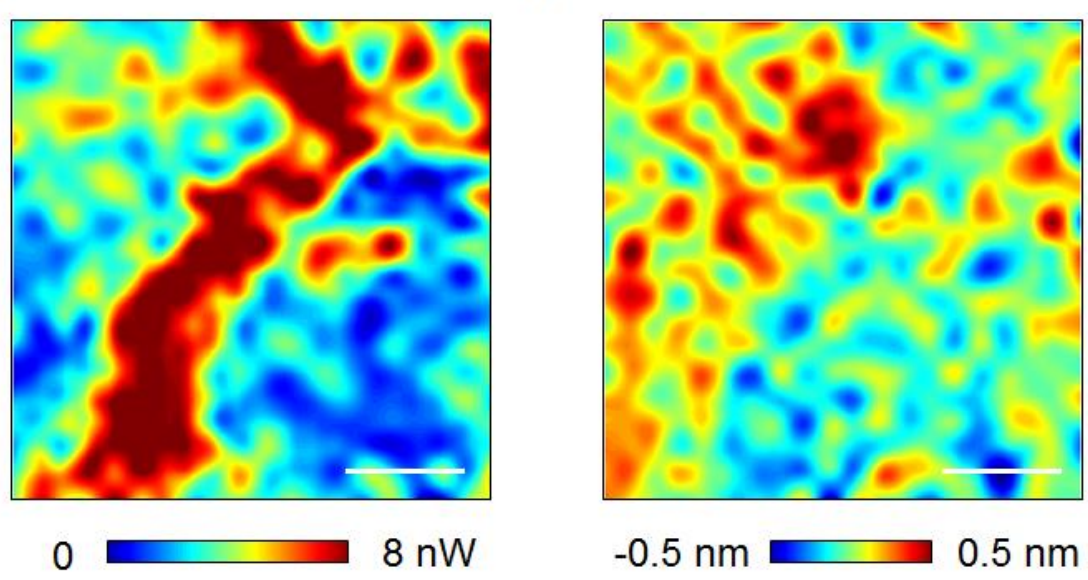

Figure 5. The imC-AFM analysis on RF-sputtered $\mathrm{Li}_{1} \mathrm{Mn}_{2} \mathrm{O}_{4}$. (a) 3D AFM morphology of the surface, note the area in the white box represents the $200 \times 200 \mathrm{~nm}^{2}$ area where $400 \mathrm{I}-\mathrm{V}$ curves are acquired (spacing $10 \mathrm{~nm}, 10 \mathrm{~V}$ bias sweep at $0.2 \mathrm{~Hz}$ ). (b) imC-AFM current map indicating the presence of two separate grains. The dotted line indicates the grain boundary. (c) Hysteresis loop area indicating anomalously high ionic activity at the grain boundary. Note the color range has been reduced to accentuate the contrast in the internal of the grains. (d) Vertical deflection changes of AFM cantilever due to local volume expansion or compression. A 20 x 20 grid with a spacing of $10 \mathrm{~nm}$ in $\mathrm{x}$ and $\mathrm{y}$ was probed with a sweep rate of $0.2 \mathrm{~Hz}$.

Therefore, at the scale of individual grains, large differences for the mobility of lithium in welldefined planar orientation of the LMO unit cell are anticipated. In addition, by looking at associated volume expansion in Figure 5d, G1 shows higher change compared to G2. The latter is measured as described in Figure $4 \mathrm{c}$ by leveraging the high sensitivity of the AFM to capture 
the vertical deflection of the cantilever (pm resolution). This indicates that significant structural changes have occurred underneath the tip during the individual voltage sweeps. In Figure 5d, the poorly lithiated grain (G1) shows relatively high ionic activity, i.e. lithium incorporation, and therefore a significant volume expansion reaching $0.5 \mathrm{~nm}$. On the contrary, the highly lithiated grain (G2) has a reduced ionic activity, thus it does not expand or even slightly compresses, possibly due to relaxation of the surface which is saturated with lithium by means of an internal galvanic process. This observation suggests that from large variations in ion mobility, the structural evolution can differ significantly from grain to grain. The strain accommodation by the grain boundary and the neighboring grains will play an important role for the variability in structural deformation, thus representing a critical parameter for the cell cyclability.[26] Note that assuming a spherical probed volume with a radius of $10 \mathrm{~nm}$, the experimental values obtained for the magnitude of the structural changes, are in line with the expectation of $4 \%$ to $6 \%$ volume expansion as reported in literature.[27] Finally, in agreement with the work of Jesse et al. using ESM,[11] a clear enhancement of the ionic activity is observed in the GB (Figure 5c). This phenomenon is most likely to occur due to the defective nature of grain boundaries. Essentially the defects lowers the activation energy of Li ions to hop from one crystal site to the next. Remarkably, the magnitude of the I-V hysteresis was measured to be 2 to 5 times larger at the grain boundary compared to the neighboring internal grain area.

\section{Experimental Section}

Synthesis:

Electrodeposited LMO:

$\mathrm{MnO}_{2}$ thin films were deposited by electrochemical deposition using a three-electrode setup where a Pt mesh is used as a counter electrode and $\mathrm{Ag} / \mathrm{AgCl} / 3 \mathrm{M} \mathrm{NaCl}$ as a reference electrode 
(BASi analytical, $0.22 \mathrm{~V}$ vs SHE). The electrolytic manganese dioxide (EMD) thin films were deposited from an aqueous solution of $0.5 \mathrm{M} \mathrm{MnSO}_{4} \cdot \mathrm{H}_{2} \mathrm{O}(98.0-101.0 \%$, Alfa easer $)$ and $0.5 \mathrm{M}$ $\mathrm{CH}_{3} \mathrm{COONa}$ (ReagentPlus, 99.0\% Sigma Aldrich) with an addition of $\sim 10 \mathrm{wt} \%$ ethanol. The EMD deposition was performed at $2.5 \mathrm{~mA} / \mathrm{cm}^{2}$ for $1 \mathrm{~s}$ as a nucleation step followed by $200 \mathrm{~s}$ at $0.5 \mathrm{~mA} / \mathrm{cm}^{2}$ for the film growth. After, the $\mathrm{EMD}\left(\mathrm{MnO}_{2}\right)$ was converted to $\mathrm{LiMn}_{2} \mathrm{O}_{4}$ by solid state reaction using a $\mathrm{Li}_{2} \mathrm{CO}_{3}$ precursor. The $\mathrm{Li}_{2} \mathrm{CO}_{3}$ layer was deposited on top of the EMD layer by chemical solution deposition. The EMD conversion to LMO was done upon a thermal annealing at $350{ }^{\circ} \mathrm{C}$ for $120 \mathrm{~min}$ in air.

\section{RF-sputtered LMO:}

A $100 \mathrm{~nm} \mathrm{LiMn} 2 \mathrm{O}_{4}$ (LMO) thin film electrode was deposited by RF-sputtering from a 4' target (Neyco, $99.5 \%$ purity). The deposition was done under Ar flow of $25 \mathrm{sccm}$ at $100 \mathrm{~W}$ of power. The chamber base pressure was kept at $\sim 3 \times 10^{-6}$ Torr. During deposition the pressure was kept at $\sim 3 \times 10^{-3}$ Torr. A post heating treatment is performed for $20 \mathrm{~min}$ at $800{ }^{\circ} \mathrm{C}$ in oxygen atmosphere at a heating rate of $10{ }^{\circ} \mathrm{C} / \mathrm{min}$ (Nabertherm RHTH 120). This annealing process helps making the LMO thin film crystalline and electrochemical active. The films were stored in an $\mathrm{Ar}$ filled glovebox $(\mathrm{O} 2, \mathrm{H} 2 \mathrm{O}<1 \mathrm{ppm})$ to avoid undesired reactions with air.

\section{$C-A F M$ and imC-AFM measurements:}

Standard C-AFM measurements are performed in IONTOF SIMS/SPM Combination tool (TOFSIMS V, ION-TOF GmbH, Münster, Germany) using conductive boron-doped full diamond tip (FDT) in contact-mode (low force) while biasing the sample. Ion modulated CAFM measurements were performed in an EnviroScope (The Digital Instruments) by sweeping in a dense grid ( $20 \mathrm{x} 20$ points, $10-20 \mathrm{~nm}$ spacing in $\mathrm{x}$ and $\mathrm{y})$ at a slow rate $(0.2 \mathrm{~Hz})$ and a stop voltage of $10 \mathrm{~V}$. Afterwards during an automated analysis in Matlab the critical parameters were extracted from the dense grid points by the numerical integration of the area between the 
subsequent DC bias sweeps. Finally, to generate a 2D map the results are linearly interpolated over the size of the scan area.

SIMS:

Experiments were carried out by means of a TOF-SIMS instrument (TOFSIMS V, ION-TOF GmbH, Münster, Germany) equipped with a $30 \mathrm{keV}$ liquid metal ion gun (LMIG) operating with bismuth primary ions. ToFSIMS chemical maps were acquired in fast imaging mode using $\mathrm{Bi}_{3}{ }^{+}$with currents of $0.3 \mathrm{pA}$ at $30 \mu \mathrm{s}$ of cycle time. Depth profiles were acquired in dual beam mode, using as sputter gun $\mathrm{O}_{2}{ }^{+} 1 \mathrm{keV}\left(400 \mathrm{x} 400 \mu \mathrm{m}^{2}\right)$ and as analysis beam $\mathrm{Bi}_{1}{ }^{+} 15 \mathrm{keV}(100$ $\mathrm{x} 100 \mu \mathrm{m}^{2}$ ) in high current bunched mode. Interpretation was carried out using Surface Lab software v6.7 (ION-TOF GmbH, Münster, Germany).

\section{Conclusion}

In summary, using $\mathrm{MnO}_{2}$-based cathodes as model systems, we have demonstrated for the first time, a new methodology that enables the electrical, structural, chemical, and electrochemical analysis of Li-ion insertion electrodes at the nanometer scale. A nanosized and biased tip is used for imaging and manipulation of lithium ions. We have probed the relationship between lithium concentration and conductivity in LMO, while the combination of C-AFM and SIMS is used to demonstrate the effective tip-induced ionic migration in confined volumes (below $1000 \mathrm{~nm}^{3}$ ). After showing how the lithium concentration can change the local conductivity under the tip, we have introduced $i m \mathrm{C}-\mathrm{AFM}$ as a method to detect the ionic activity and volume changes corresponding to lithiation and delithiation in thin films with ca. $20 \mathrm{~nm}$ resolution. The local electrochemical properties of electrodeposited and RF-sputtered LMO have been investigated comparing crystalline grains with GBs, for which an anomalously high ionic diffusion coefficient was observed. In contrast with other modes, imC-AFM aims to improve the sensitivity and reliability of the measurement by using multiple response factors, i.e. local conductivity variations and tip vertical deflection. For this reason, our concept can be applied 
well beyond $\mathrm{Li}$-ion insertion electrodes to other mixed electronic-ionic conductors. Moreover, it is ideal to screen new material systems with unknown electrical properties or expansion (Vegard) coefficients. For their technological importance in future battery applications, our research can therefore boost the development and commercialization of microbatteries and ASB.

\section{Conflicts of interest}

There are no conflicts to declare

\section{Acknowledgements}

A. Sepúlveda would like to acknowledge the EU Horizon 2020 research and innovation program under the MSCA grant agreement No 658057. This project has received funding from the European Union's Horizon 2020 research and innovation program under grant agreement No 688225 (Metro4-3D - Metrology for future 3D-technologies).

\section{References}

[1] J. Janek, W.G. Zeier, Nat. Energy, 2016, 1, 16141.

[2] A.A. Talin, D. Ruzmetov, A. Kolmakov, K. McKelvey, N. Ware, F. El Gabaly,B.

Dunn, H. S. White, Fabrication, ACS Appl. Mater. Interfaces, 2016, 8, 32385.

[3] P. M. Vereecken, C. Huyghebaert, ECS Transactions, 2013, 58, 111.

[4] M. V. Reddy, B. Pecquenard, P. Vinatier, A. Levasseur, Electrochem. Commun., 2007, 9, 409.

[5] J. Lu, T. Wu, K. Amine, Nat. Energy., 2017, 2, 1701.

[6] S. Yang, B. Yan, L. Lu, K. Zeng, RSC Adv., 2016, 6, 94000.

[7] S. Guo, S. Jesse, S. Kalnaus, N. Balke, C. Daniel, S. V. Kalinin, J. Electrochem. Soc., 2011, 158, A982.

[8] E. Strelcov, Y. Kim, S. Jesse, Y. Cao, I.N. Ivanov, I.I. Kravchenko, C. Wang, Y. Teng, L. Chen, Y.H. Chu, S. V. Kalinin, Nano Lett., 2013, 13, 3455. 
[9] J. Zhu, L. Lu, K. Zeng, ACS Nano., 2013, 7, 1666.

[10] E. Strelcov, S.M. Yang, S. Jesse, N. Balke, R.K. Vasudevan, S. V. Kalinin, Nanoscale, 2016, 8, 13838.

[11] S. Jesse, N. Balke, E. Eliseev, A. Tselev, N.J. Dudney, A.N. Morozovska, S. V. Kalinin, ACS Nano, 2011, 5, 9682.

[12] S. Jesse, S. V Kalinin, J. Phys. D: Appl. Phys., 2011, 44, 464006.

[13] J. Maier, Nat. Mater., 2005, 4, 805.

[14] X. Zhang, X. Cheng, Q. Zhang, J. Energy Chem., 2016, 25, 967.

[15] B. Put, P.M. Vereecken, N. Labyedh, A. Sepulveda, C. Huyghebaert, I.P. Radu, A. Stesmans, ACS Appl. Mater. Interfaces., 2015, 7, 22413.

[16] P. van der Heide, Secondary ion mass spectrometry an introduction to principles and practices, Wiley, Hoboken, NJ, USA, 2014.

[17] A. Kumar, F. Ciucci, A.N. Morozovska, S. V Kalinin, S. Jesse, Nat. Chem., 2011, 3, 707.

[18] A. V. Ievlev, P. Maksymovych, M. Trassin, J. Seidel, R. Ramesh, S. V. Kalinin, O. S. Ovchinnikova, ACS Appl. Mater. Interfaces., 2016, 8, 29588.

[19] A. Schulze, PhD thesis, KU Leuven, April, 2013.

[20] M.G. Verde, L. Baggetto, N. Balke, G.M. Veith, J.K. Seo, Z. Wang, Y. S. Meng, ACS Nano, 2016, 10, 4312.

[21] A. Moradpour, O. Schneegans, S. Franger, A. Revcolevschi, R. Salot, P. AubanSenzier, C. Pasquier, E. Svoukis, J. Giapintzakis, O. Dragos, V. Ciomaga, P. Chrétien, Adv. Mater., 2011, 23, 4141.

[22] I. Uchida, S. Yamamura, H. Koshika, M. Nishizawa, T. Matsue, I. Uchida, J. Solid State Electrochem., 1998, 2, 211.

[23] N. Balke, S. Jesse, Y. Kim, L. Adamczyk, A. Tselev, I.N. Ivanov, N. J. Dudney, S. V. Kalinin, Nano Lett., 2010, 10, 3420. 
[24] M.M. Thackeray, W.I.F. David, P.G. Bruce, J.B. Goodenough, Mater. Res. Bull., 1983, 18, 461 .

[25] F. Mattelaer, P.M. Vereecken, J. Dendooven, C. Detavernier, Chem. Mater., 2015, 27, 3628.

[26] H. Wang, Y. Jang, Y. Chiang, Electrochem. Solid-State Lett., 1999, 2, 490.

[27] K. Kuriyama, A. Onoue, Y. Yuasa, K. Kushida, Surf. Sci., 2007, 601, 2256. 